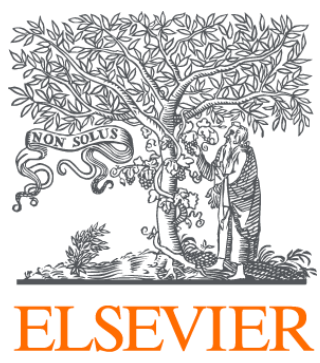

Since January 2020 Elsevier has created a COVID-19 resource centre with free information in English and Mandarin on the novel coronavirus COVID-

19. The COVID-19 resource centre is hosted on Elsevier Connect, the company's public news and information website.

Elsevier hereby grants permission to make all its COVID-19-related research that is available on the COVID-19 resource centre - including this research content - immediately available in PubMed Central and other publicly funded repositories, such as the WHO COVID database with rights for unrestricted research re-use and analyses in any form or by any means with acknowledgement of the original source. These permissions are granted for free by Elsevier for as long as the COVID-19 resource centre remains active. 


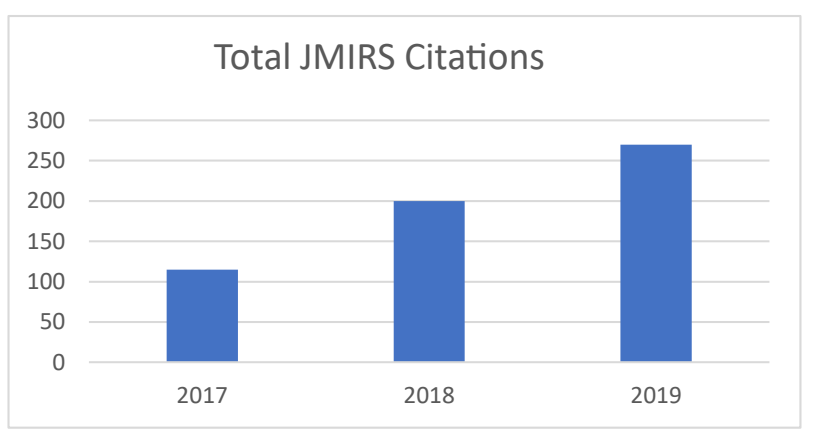

Figure 2. Total JMIRS citations from items published between 2008-2019; data extracted from Scopus.

downloads per month. With the inclusion of our journal in MEDLINE in late 2018, we are looking forward to seeing the JMIRS continue to flourish; although it takes 2-3 years to see the full effect of indexing because papers that will cite JMIRS content are still being written, going through peer review and then the publication process. However, we are already seeing an increase, with 115 citations already recorded in 2020 as of this writing (see Figure 2).

Although the deadline for our 2020 special edition on "Soft Skills" has passed, there is still time to submit a Letter to the Editor or Editorial. The editors will be looking for let- ters that offer reflections, insights, and creative suggestions related to the topic from all disciplines. Please submit by August 17, 2020; if you have questions, please contact us at editor@camrt.ca. I am looking forward to seeing this issue come together with our Guest Editor, Sue Robins. Sue is a writer, patient advocate and breast cancer survivor, and has contributed to JMIRS previously as an author.

Our special issue for 2021 is education. We welcome submissions about any aspect of MRT education, undergraduate, graduate, patient, public, continuing professional development and more. We've picked this topic early to allow more time for research and writing based on feedback from our readership. Stay tuned for more details and a submission date!

Our journal exists to encourage the creation, collection and dissemination of new knowledge in our field. We're here to help, so please reach out if you have questions about your writing, want more information on the publication process, if you're interested in reviewing or if you're wondering if your topic is suitable for publication. You can tweet your comments to our journal account @JMIRS1, or email me directly at editor@camrt.ca to have your feedback published in our next edition.

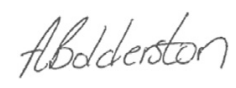

\title{
Message de la rédactrice en chef
}

\author{
Amanda Bolderston, FACTRM, MSc, EdD
}

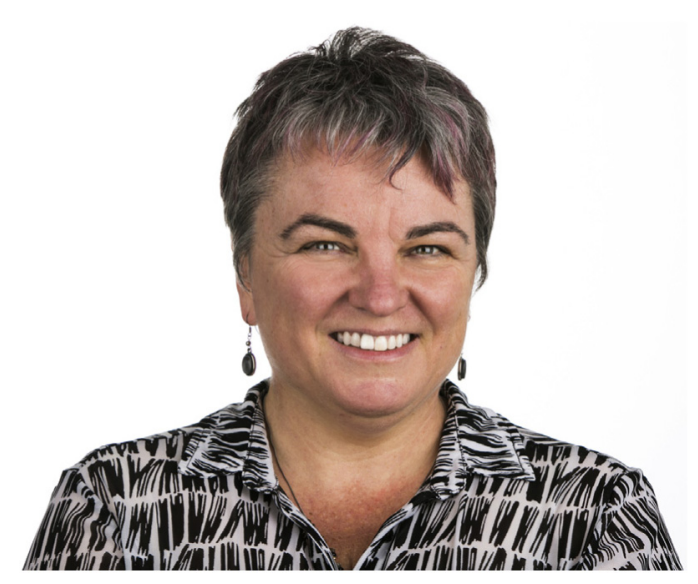

Bienvenue à notre numéro de l'été. Au moment où j'écris ceci, plusieurs TRM de première ligne sont confrontés à des pressions sans précédent en raison de l'éclosion de COVID-
19. À titre de travailleurs essentiels, ils et elles doivent jongler avec les soins des enfants à la maison, les inquiétudes à propos de la famille et des amis et l'anxiété de travailler dans un milieu en rapide évolution. Nos patients font face à des retards dans le dépistage, le traitement et autres procédures d'imagerie et services médicaux tandis que nous donnons la priorité aux personnes malades et présentant un degré de risques élevé. D’autres TRM font du dépistage et aident en arrière-scène, y compris le personnel administratif et enseignant (dont certains sont sortis de la retraite pour intervenir à titre bénévole). Nos étudiants font face à des perturbations et à des retards importants dans leurs cours, ou entrent dans un milieu de travail transformé plus rapidement que prévu. Le corps professoral universitaire enseigne et prépare les cours en ligne. Nous sommes tous à la recherche d'information pour nous aider à composer avec la situation et le JIMSR s'est adapté en trouvant des façons de raccourcir le cycle de publication. Par exemple, nous publions des renseignements utiles sur la COVID-19 sous la forme de lettres à la rédaction (qui peuvent être mises en ligne beaucoup plus rapidement 


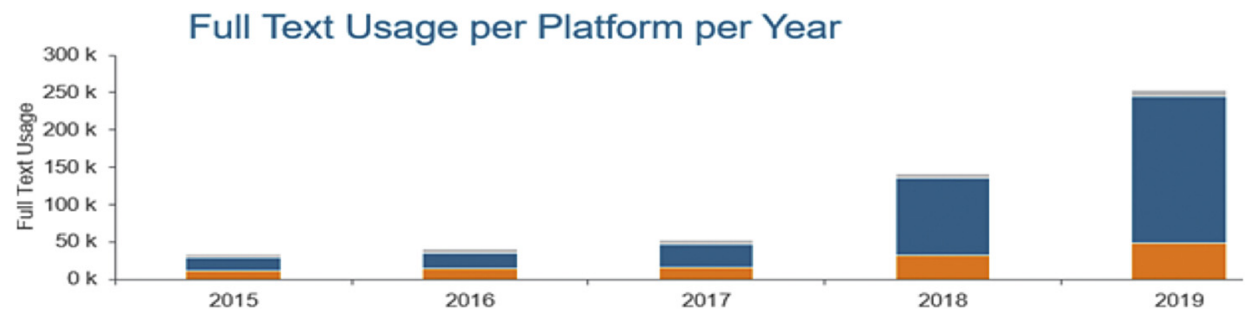

Figure 1. Utilisation en plein texte par plateforme par année. SD, ScienceDirect (orange); JBS, Journal Based Solutions (bleu); CK, Clinical Key (gris). L'utilisation d'articles en plein texte est la somme des téléchargements en PDF et des consultations en format HTML.

que les propositions d'articles traditionnelles). Comme d'habitude, ceci est le résultat du travail de nos lecteurs critiques et de nos rédacteurs en chef adjoints, qui ont accepté une charge de travail supplémentaire. Nous souhaitons les remercier, ainsi que tous les TRM, pour leurs efforts incessants pendant cette pandémie.

Le podcast est une autre des méthodes de diffusion non traditionnelle que nous explorons. Nous enregistrons maintenant un podcast pour chaque numéro, dans lequel nous discutons avec l'un des auteurs à propos de ses recherches. C'est une façon rapide (10-15 minutes) de découvrir l'article choisi sous un nouvel angle. Pour notre premier podcast, nous parlons avec la professeure Phyllis Butow de l'Université de Sydney, l'une des auteurs de notre article vedette de mars, «Unmasking anxiety: A qualitative investigation of health professionals' perspectives of mask anxiety in head and neck cancer $\gg$. Vous pouvez le trouver sur Apple Podcasts, Spotify et plusieurs autres plateformes, ou sur notre site Web à JMIRS.org.

Avec Carly, notre directrice de la rédaction, j'ai entrepris de préparer un rapport pour le conseil d'administration de l'ACTRM afin de souligner les réalisations du Journal jusqu'à la fin de 2019. Nous avons une diffusion plus large que jamais, les mesures indiquant une croissance sur toutes les plateformes-le contenu du journal est disponible à partir de notre page d'accueil à jmirs.org (JBS), sur Science Direct (SD) et sur Clinical Key (CK). En 2019, notre plateforme JBS a enregistré 194410 consultations d'articles en plein texte (augmentation de 73\% sur 2018, voir Figure 1); 143 237 pages consultées (augmentation de 42\%); et 69899 visiteurs uniques (augmentation de 83\%). Les téléchargements à partir de SD ont augmenté de 39972 en 2018 à 56628

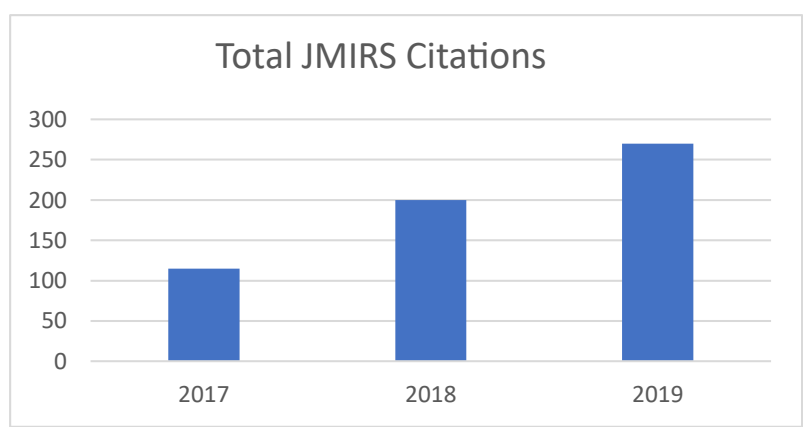

Figure 2. Nombre total de citations du JIMSR pour des articles publiés entre 2008 et 2019; données extraites de Scopus. en 2019 (augmentation de 41\%), pour une moyenne de 4 747 téléchargements par mois. Avec l'inclusion de notre Journal dans Medline à la fin de 2018, nous prévoyons que le JIMSR continuera de progresser; bien qu'il faille habituellement entre deux et trois ans pour voir l'effet de l'indexation, parce que les articles qui citeront le contenu du JIMSR doivent encore passer par l'étape de la rédaction, de la révision par les pairs et du processus de publication. Nous voyons quand même déjà une augmentation, avec 115 citations déjà enregistrées jusqu'ici en 2020 (voir Figure 2).

Bien que la date limite pour notre numéro spécial de 2020 sur les compétences humaines soit passée, il est encore temps de nous faire parvenir une lettre à la rédaction ou un éditorial. La rédaction recherche des lettres qui présentent des réflexions, des idées et des suggestions sur la question dans toutes les disciplines. La date limite est le 17 août 2020; si vous avez des questions, veuillez communiquer avec nous par courriel à editor@camrt.ca. Je prépare ce numéro avec Sue Robins, notre rédactrice en chef invitée. Auteure, militante des droits des patients et survivante du cancer du sein, Sue a publié dans le Journal dans le passé.

Notre numéro spécial de 2021 portera sur l'éducation. Nous souhaitons obtenir des propositions d'articles sur tous les aspects de l'éducation des TRM, la formation de premier cycle et la formation supérieure, l'éducation des patients et du public, le perfectionnement professionnel continu et autres. Nous avons choisi le sujet tôt afin de donner plus de temps pour la recherche et la rédaction, en réponse aux commentaires de notre lectorat. Des renseignements plus détaillés et l'annonce de la date limite suivront!

Notre journal existe pour encourager la création, la collecte et la diffusion de nouvelles connaissances dans notre domaine. Nous sommes là pour vous aider, alors n'hésitez pas à communiquer avec nous si vous avez des questions sur la rédaction, si vous avez besoin de plus d'information sur le processus de publication, si vous souhaitez offrir vos services comme lecteur ou lectrice critique ou simplement si vous vous demandez si votre sujet est propice à la publication. Vous pouvez nous envoyer un gazouillis à @JMIRS1, ou m’envoyer directement un courriel à editor@camrt.ca pour que vos commentaires soient publiés dans le prochain numéro.

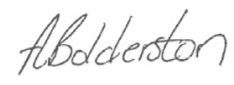

